Review

\title{
Plant responses to extracellular nucleotides: Cellular processes and biological effects
}

\author{
Collene R. Jeter ${ }^{1} \&$ Stanley J. Roux ${ }^{2}$ \\ ${ }^{1}$ Science Park-Research Division, Department of Carcinogenesis, The University of Texas MD Anderson Cancer Center, \\ Smithville, TX, 78957, USA, ${ }^{2}$ Section of Molecular Cell and Developmental Biology, The University of Texas-Austin, \\ Austin TX 78712-0183, USA
}

Received 1 July 2005; accepted in revised form 26 August 2005; Published online 11 August 2006

Key words: apyrase, calcium signaling, reactive oxygen species, stress-regulated genes, wound responses

\begin{abstract}
Higher plants exhibit cellular responsiveness to the exogenous application of purine nucleotides in a manner consistent with a cell-cell signaling function for these molecules. Like animals, plants respond to extracellular ATP, ADP, and stable analogues (e.g., ATP $\gamma \mathrm{S}$ and ADP $\beta S$ ) by increasing the cytoplasmic concentration of calcium. Agonist substrate specificity and concentration dependency suggest receptor mediation of these events, and, although the identity of the plant receptor is currently unknown, pharmacological analysis points to the involvement of a plasma membrane-localized calcium channel. Extracellular ATP can also induce the production of reactive oxygen species and stimulate an increase in the mRNA levels of a number of stress- and calcium-regulated genes, suggesting a role for nucleotide-based signaling in plant wound and defense responses. Furthermore, the growth and development of plants can also be altered by the application of external ATP. Recent studies are only beginning to uncover the complexities of plant signaling networks activated in response to extracellular ATP and how these might interact to affect plant physiological processes.
\end{abstract}

\section{Introduction}

Extracellular signaling functions for adenosine nucleoside tri- and diphosphates have been well documented in animals for over 50 years. Early observations of nucleotide effects on plant biological processes suggest that extracellular ATP could be a signaling agent in plants as well. For example, these reports show that exogenous application of ATP increases Lilly pollen tube mitotic activity [1] modulates stomatal guard cell aperture [2], and promotes the closure of the Venus fly trap [3]. Externally applied caffeine, another purine derivative, induces pronounced inhibitory effects on plant developmental and cellular processes, including sieve plate formation [4], tracheary element differentiation [5] and pollen tube growth [6]. Importantly, cytokinins, which are adenine derivatives, are among the major classes of plant hormones regulating plant growth and development [7].

Although cytokinin has been accepted as a bona fide extracellular signaling agent in plants, further evidence of a cell-cell signaling function for extracellular ATP has been required to circumvent arguments that its effects are simply due to alterations in the cells' energy status. The presence of prospective ATP release mechanisms has supported the

Correspondence to: Stanley J. Roux, Section of Molecular Cell and Developmental Biology, The University of Texas-Austin, University Station A6700, Austin, TX 78712-0183, USA. Tel: +1-512-4714238; Fax: +1-512-2323402; E-mail: sroux@uts.cc.utexas.edu notion that ATP is present in the plant extracellular matrix (ECM), thereby satisfying the requirement for an endogenous signal source. Cell lysis is a passive means by which cytoplasmic ATP could exit any cell, and plant cells are undoubtedly subject to this simplistic mechanism for ATP release during events such as wounding or herbivory. Exocytosis of secretory vesicles containing ATP [8], as well as efflux through anion channels $[9,10]$ or in association with Multidrug Resistance (MDR) Transporters [11] all represent potential ATP efflux mechanisms characterized in animals, and similar mechanisms are possible in plants as well. In this regard, expression of at least one plant MDR transporter homologue, AtPGP1 (Arabidopsis thaliana $p$-glycoprotein) in yeast has been shown to increase ATP release into the growth medium, and to similarly increase extracellular ATP concentrations when overexpressed in transgenic Arabidopsis [12].

The conservation of key regulatory players further substantiates a putative signaling function for extracellular ATP in the plant ECM. ATP/ADP hydrolytic activity could participate in the termination of an ATP signal, the maintenance of responsiveness to such a signal, and in the recycling of the ATP constituent components. For example, numerous apyrase (NTPDase) homologues, at least some of which have been shown to have an extracellular localization, have been cloned from a wide variety of plants including Solanum tuberosum [13], Pisum sativum [14], Dolichos biflorus [15] and Arabidopsis thaliana [16], among others [17, 18]. The NTP/NDP 
hydrolyzing activity of these enzymes, presumably along with the action of nucleotidases and phosphatases, has been proposed to participate in phosphate scavenging in plants [19] and the maintenance of xenobiotic resistance in association with MDR transporters [12]. Of particular interest, apyrases have been implicated in a variety of developmental processes as well, including pollen germination [20], nodulation [21, 22] and growth (Sun, Steinebrunner and Roux, unpublished).

\section{Rapid cellular responses to external application of purine nucleotides: Secondary messengers and signaling mechanisms}

Regardless of these observations, definitive evidence of a cell-cell signaling function for extracellular ATP would require measurements of a rapid biological response. At the cellular level, changes in plasma membrane potential and/ or the induction of secondary messengers would demonstrate that plant cells do indeed have the ability to directly perceive and respond to extracellular ATP. To this end, Lew and Dearnaley [23] were the first to evaluate the effects of external application of a variety of nucleoside triand diphosphates on the membrane potential of Arabidopsis root hair cells. The authors reported $1 \mathrm{mM}$ ATP or ADP, as well as GTP were consistently capable of inducing strong membrane depolarizations, whereas TTP and CTP, as well as free phosphate were ineffective (summarized in Table 1). Titrations of root hair responses to ATP and ADP suggested concentration dependence and a half-maximal response for ATP at 440 versus $10 \mu \mathrm{M}$ for ADP [23] (Table 1). Substrate specificity and concentration dependency pointed to the existence of a distinct receptor, or receptors, for extracellular ATP and ADP.

Although Lew and Dearnaley considered the possibility that extracellular ATP or ADP could increase the cytoplasmic calcium ion concentration $\left(\left[\mathrm{Ca}^{2+}\right]_{\mathrm{cyt}}\right)$, a common intracellular signaling agent induced by nucleotide-based signaling mechanisms in animals and a central signaling paradigm in plants, the authors reported no detectable alterations in the $\left[\mathrm{Ca}^{2+}\right]$ in root hairs. A wide variety of physiological stimuli, including light, environmental stresses, hormonal signaling and, in leguminous plants, nodulation factors secreted by nitrogen-fixing rhizobial bacteria induce an increase in $\left[\mathrm{Ca}^{2+}\right]_{\text {cyt }}[24,25]$. Of particular importance, wounding, an obvious potential source of ATP release, induces a calcium wave in Arabidopsis leaves [26]. This study made use of transgenic Arabidopsis
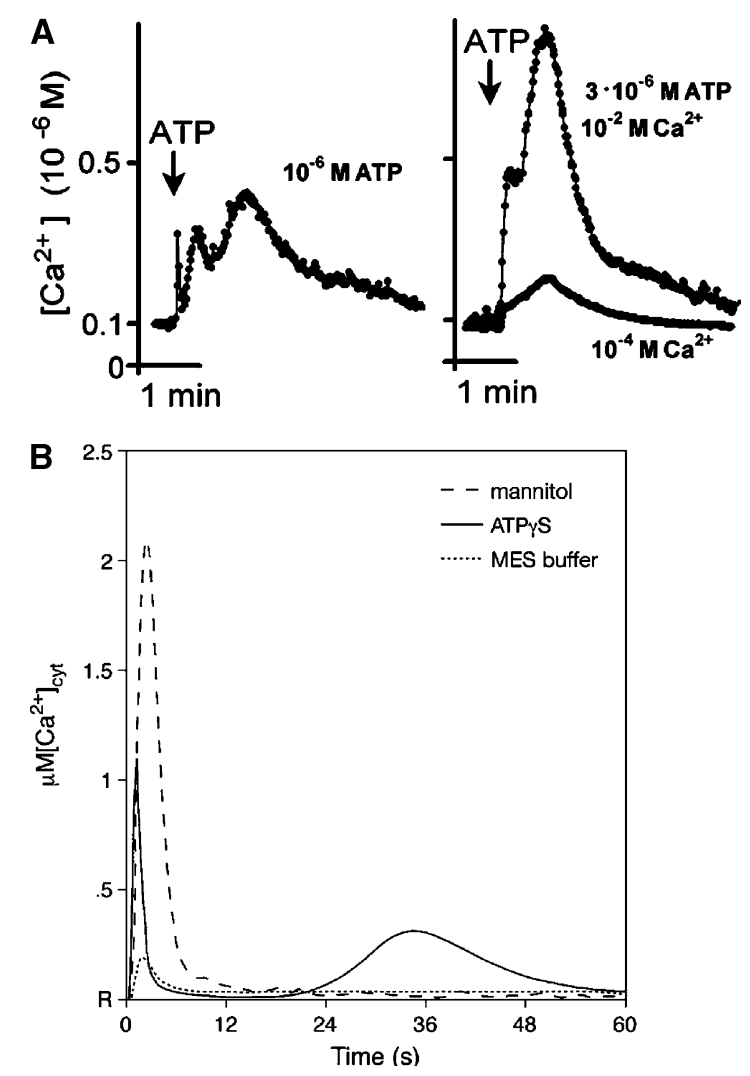

Figure 1. Transgenic apo-aequorin-expressing Arabidopsis display calcium transients in response to exogenously applied ATP. A Typical response of excised roots to $3 \mu \mathrm{M}$ ATP, measured in a recording solution containing either 10 or $0.1 \mathrm{mM} \mathrm{CaCl}_{2}, 2 \mathrm{mM} \mathrm{MES}$, pH 5.7 and $4 \mu \mathrm{g} / \mathrm{ml}$ of the coelenterazine luminophore cofactor. B Typical response of whole seedlings to $500 \mu \mathrm{M}$ ATP, $50 \mathrm{mM}$ MES (buffer only), and $0.666 \mathrm{M}$ mannitol. $R$ indicates resting calcium levels. (From Demidchik et al. [27] and Jeter et al. [29].)

expressing aequorin, a highly sensitive bioluminescent calcium reporter system.

To further evaluate the extracellular signaling activities of nucleotide derivatives, two independent studies utilizing aequorin-expressing Arabidopsis were performed nearly in parallel, one focusing on roots [27] and the other on whole seedlings [28, 29]. These results have verified that exogenous application of either ATP, ADP, or, importantly, a variety of poorly hydrolysable stable analogs can indeed significantly increase the $\left[\mathrm{Ca}^{2+}\right]$ in plant cells. Demidchik et al. [27] utilized excised roots from adult plants bathed in a recording solution containing $10 \mathrm{mM}$ $\mathrm{CaCl}_{2}, 2 \mathrm{mM}$ MES and Tris buffered to $\mathrm{pH} 5.7$ and nicely demonstrated the induction of a strong calcium transient (up to $\mu \mathrm{M}$ levels, compared to resting levels of approx-

Table 1. Effects of applied purine nucleotides on different plant processes.

\begin{tabular}{|c|c|c|c|c|c|c|}
\hline Tissue & Agonists & $\begin{array}{c}\text { Range of } \\
\text { [nucleotide] }\end{array}$ & Second messenger & Antagonist & Effect on whole plant & Reference \\
\hline Root hair & ATP, ADP, GTP & $\mu \mathrm{M}-\mathrm{mM}$ & $\begin{array}{l}\text { Membrane } \\
\text { depolarization }\end{array}$ & N.d. & Root hair growth & 23 \\
\hline Root & ATP, ADP, 2MeATP & $\mathrm{nM}-\mu \mathrm{M}$ & Calcium & $\mathrm{Gd}^{3+}$, PPADS, suramin & N.d. & 27 \\
\hline Seedling & $\mathrm{ATP} \gamma \mathrm{S}, \mathrm{ATP}, \mathrm{ADP} \beta \mathrm{S}$ & $\mu \mathrm{M}-\mathrm{mM}$ & Calcium & $\mathrm{La}^{3+}, \mathrm{Gd}^{3+}$, BAPTA & Stress-related gene changes & 29 \\
\hline Leaf & ATP & $\mathrm{nM}-\mu \mathrm{M}$ & ROS & BAPTA, PPADS, $\mathrm{La}^{3+}$ & Wound-related gene changes & 32 \\
\hline
\end{tabular}


imately $100 \mathrm{nM}$ ) in response to agonist concentrations ranging from $\mu \mathrm{M}$ to $\mathrm{mM}$ (Figure $1 \mathrm{~A}$ ). They also documented the substrate specificity of the response (e.g., the application of UTP had minimal effects, and AMP was ineffective). Furthermore, in spite of the heterogenous nature of the tissue responding, the plotted response curves revealed dose-dependency and an apparent saturation of the response, suggesting receptor mediation [27]. Pharmacological analysis with potential antagonists also showed that the response was sensitive to the calcium channel blocker gadolinium $\left(\mathrm{Gd}^{3+}\right)$, as well as the P2-receptor antagonists suramin and PPADS (pyridoxal phosphate-6azo-(benzene-2,4-disulfonic acid)) (Figure 2A). The authors interpreted these effects as being consistent with an influx of calcium from the plant ECM through a channel in the plasma membrane.

Jeter et al. [29] substantiated the data of Demidchik et al. [27], showing not only an increase of $\left[\mathrm{Ca}^{2+}\right]_{\text {cyt }}$ in response
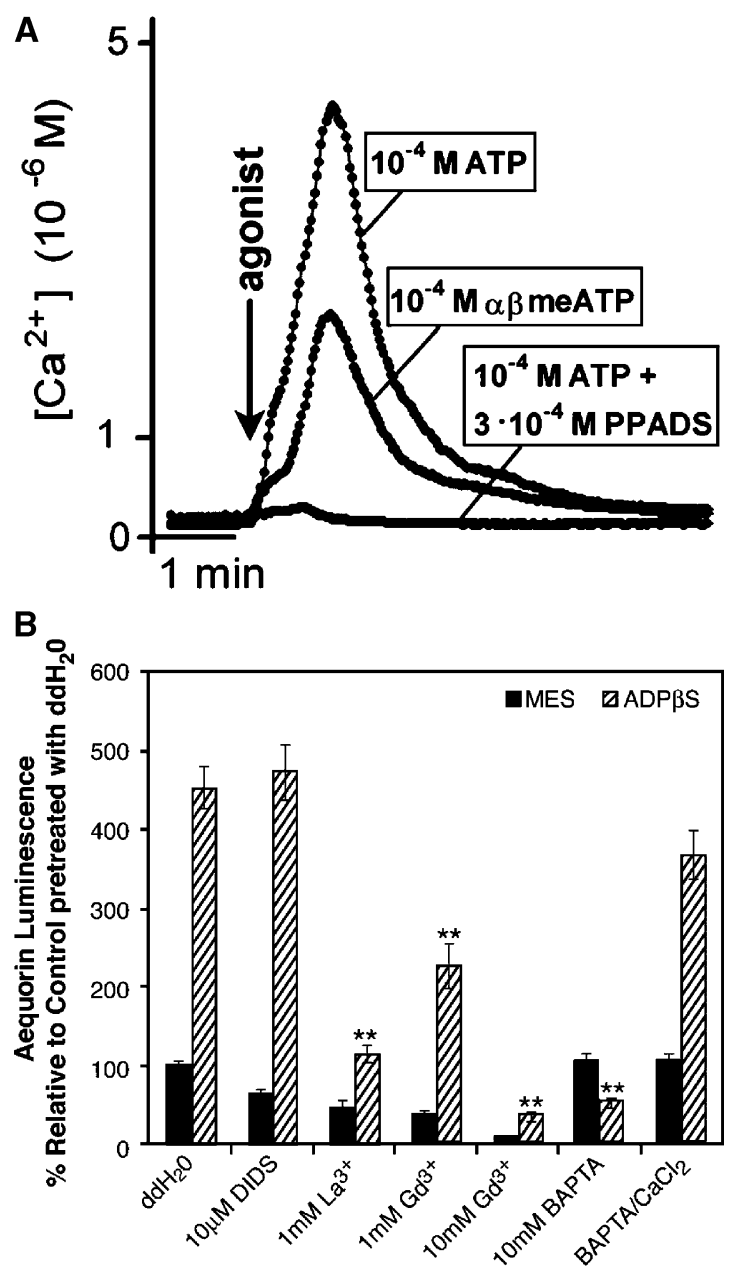

Figure 2. Antagonists can inhibit the increase in $\left[\mathrm{Ca}^{2+}\right]_{\text {cyt }}$ induced by purine derivatives. A The P2-receptor antagonist PPADS $(0.3 \mathrm{mM})$ blocks the response of roots to $100 \mu \mathrm{M}$ ATP. A representative trace is shown. B Pretreatment of aequorin seedlings with calcium channel blockers or the calcium chelator BAPTA inhibit the whole seedling luminescent response to $500 \mu \mathrm{M}$ ADP $\beta S$, as compared to pretreatment with water $\left(\mathrm{ddH}_{2} \mathrm{O}\right)$. Groups consist of a minimum of six seedlings/group, error bars indicate \pm $\mathrm{SE}$, and double asterisk indicate $P<0.01$ ). (From Demidchik et al [27] and Jeter et al [29].) to the stable thiol derivatives ATP $\gamma \mathrm{S}, \mathrm{ADP} \beta \mathrm{S}$, but also a calcium dependency for downstream changes in gene expression. They tested whole 7-day-old Arabidopsis seedlings directly grown in 96-well plates for calcium flux responsiveness to ATP $\gamma \mathrm{S}, \mathrm{ADP} \beta \mathrm{S}$ and AMPS (buffered at $\mathrm{pH} 5.7$ with $50 \mathrm{mM}$ MES) with the root anchored in a small volume of standard growth media. Like Demidchik et al., they used aequorin-expressing transgenic plants to monitor the calcium fluxes, and like those authors they recorded a biphasic response, with a rapid response peak of increased $\left[\mathrm{Ca}^{2+}\right]_{\text {cyt }}$ occurring on the order of seconds and a second peak occurring within 1 min later [27, 29] (Figure $1 \mathrm{~A}$ and $\mathrm{B}$, respectively).

However in the whole seedling system, higher agonist concentrations (ranging from $100 \mu \mathrm{M}$ to $1 \mathrm{mM}$ ) were required to stimulate a lower level peak calcium response (approximately averaging $300 \mathrm{nM}$ in response to $500 \mu \mathrm{M}$ of either ATP $\gamma \mathrm{S}$ or ADP $\beta S$ ) as compared to the data of Demidchik et al., with a significant portion of the signal coming from the aerial (shoot) portion of the seedling (comparison summarized in Table 1). It is unknown whether these differences should be attributed to different percentages of responding cells between the two tissues, methodological variations (the whole seedling system notably containing 100 -fold less calcium in the bathing medium than was present in the excised root system utilized by Demidchik et al.), or inherent differences in the permeability or cellular responsiveness of the two tissues to the experimental solutions.

Jeter et al. further showed that extracellular ATP and ADP analogs can enhance the increase in $\left[\mathrm{Ca}^{2+}\right]_{\text {cyt }}$ induced by oligogalacturonic acid (OGA), cell wall molecules released by wounding and previously shown to induce calcium transients in tobacco suspension cultured cells [30]. Additionally, they found that calcium channel blockers and importantly the calcium chelator BAPTA (1,2-bis(o-aminophoxy)ethane- $N, N, N^{\prime}, N^{\prime}$-tetraacetic acid) inhibit the ADP $\beta S$-induced increase in $\left[\mathrm{Ca}^{2+}\right]_{\text {cyt }}$, (Figure 2B) as well as downstream changes in gene expression, thus providing evidence that the ECM is the source of calcium ions for the nucleotide-induced increase in $\left[\mathrm{Ca}^{2+}\right]_{\text {cyt }}$ and, that these induced ionic changes are needed for the later changes in message abundance [29].

Reactive Oxygen Species (ROS) such as superoxide are produced by specialized cells of the immune system in animals in response to extracellular ATP, and this secondary messenger is also induced in plants in response to environmental stresses such as wounding and pathogen invasion [31]. Song et al. have reported that the pressure infiltration of low $\mu \mathrm{M}$ levels of ATP into mature Arabidopsis leaves can similarly stimulate the production of ROS in a dose-dependent and substrate-specific fashion [32]. The observation that this response is attenuated by calcium channel blockers, the calcium chelator BAPTA and calmodulin inhibitors places calcium signaling upstream in the transduction pathway. Of particular interest, pretreatment with adenosine can also reduce the level of ROS generated in response to extracellular ATP, suggesting the existence of feedback inhibitory processes [32]. 
A proposed biological role in mediating plant responses to abiotic and biotic stresses

Increased $\left[\mathrm{Ca}^{2+}\right]_{c y t}$ and the production of ROS are important intracellular signaling mechanisms activated as a result of environmental stresses. Abiotic stimuli including touch, wounding and osmotic stresses have all been observed to induce calcium transients $[24,26]$, and nucleotide-based signaling is essential for animal cell responses to similar forms of stress [33]. To address the possibility that extracellular ATP (or ADP) is a mediator of stress responses in plants, Jeter and colleagues evaluated the effects of these agonists on gene expression profiles of a number of genes upregulated by stress.

The levels of transcripts encoding certain members of the mitogen-activated protein kinase (MAPK) family are increased in response to touch or osmotic stress [34] as well as wounding [35]. Likewise, the mRNA levels encoding mitogen-activated protein kinase kinase kinase 1 (ATMEKK1) and mitogen-activated protein kinase 3 (ATMPK3), as well as ribosomal protein S6 kinase (ATPK19) are upregulated by both extracellular ATP and ADP, with remarkably similar kinetics as observed in response to environmental stresses [29].

Levels of ethylene $\left(\mathrm{H}_{2} \mathrm{C}=\mathrm{CH}_{2}\right)$, another major plant hormone, rise in response to stress insults [36], driven by stress-induced increases in the expression of a critical ethylene biosynthetic enzyme 1-aminocyclopropane-1carboxylic acid synthase (ACS) and of downstream ethylene responsive transcription factors (ERFs). Jeter et al. found that extracellular ATP induced increased abundance of transcripts encoding ACS6 and ERFs 2, 3, and 4. Notably, pretreatment of seedlings with the calcium channel blocker $\mathrm{Gd}^{3+}[29]$ and BAPTA (data not shown) reduced the upregulation of MAPK family members in response to $500 \mu \mathrm{M}$ ATP and inhibited the increase in ERF4 mRNA levels.

Plants are also subject to biotic stresses, and herbivory or pathogen invasion can induce a specific physiological protective mechanism termed the defense response. The oxidative burst is an important mediator of these responses, and downstream changes in gene expression alter the physiology of plants to protect against further damage or infection. Song et al. [32] observed changes in gene expression in response to infiltration of low $\mu \mathrm{M}$ levels of ATP, including increases in message abundance not only for ACS6 but also for phenylalanine ammonia lyase (PAL) 1 and lipoxygenase (LOX) 2, two other enzymes typically induced by wounding [35]. LOX2 has been implicated in the biosynthesis of a plant prostaglandin-like stress responsive hormone jasmonic acid, an important component of signaling mechanisms transducing plant cellular responses to wounding and herbivory [35].

The experimental evidence so far supports a role for extracellular ATP signaling in the regulation of plant responses to environmental stresses such as wounding and infection with pathogenic organisms. Considering the numerous reports of apyrase association with nodulation [17, $18,21,22]$ it is tempting to speculate a role for extracellular ATP in mediating the interactions of leguminous plants with potential rhizobial symbionts as well. Previous observations have noted a positive correlation between the presence of soil microflora and the [ATP] in soil water filtrates [19], suggesting rhizobia may serve as a source of ATP near the root hair infection site. Indeed, McCalvin and Stacey [22] proposed extracellular ATP may fine tune root hair responsiveness to Nod factors, including both stimulatory (e.g. calcium oscillations) and inhibitory (e.g. ethylene) effects on nodulation. However, additional studies will be required to evaluate this intriguing possibility.

\section{Biological effects of extracellular ATP on plant growth and development}

Both superoxide and ethylene are growth regulators, and the fact that applied ATP induces an increase in the production of superoxide and in the abundance of mRNA for enzymes that catalyze ethylene production, raises the question of whether extracellular ATP helps regulate growth. Tang et al. [37] tested this hypothesis and found that the answer to this question was yes. The roots of plants grown in a medium supplemented with $1 \mathrm{mM}$ ATP have impaired gravitropism, growing horizontally instead of downward; $2 \mathrm{mM}$ ATP induces root curling; and $3 \mathrm{mM}$ ATP inhibits root elongation and stimulates lateral root growth. Control experiments showed that these effects could not be mimicked by AMP or phosphate, and could not be attributed to $\mathrm{pH}$ changes or to the ability of ATP to chelate divalent cations. The hormone auxin regulates diverse aspects of root growth, and tests revealed that higher levels of applied ATP could inhibit the transport of auxin. The authors concluded that the inhibitory effects of extracellular ATP on auxin distribution could account for much of its effects on root growth [37].

The mM levels of ATP needed to induce growth effects seemed too high to be physiologically relevant. Follow-up studies showed that ATP $\gamma \mathrm{S}$ could induce the same effects as ATP, but at 10-to-20-fold lower concentrations [37, 38]. These results suggest that the action of phosphatases and apyrases known to be present in plant cell walls could reduce the effective concentration of applied ATP. The activity of these and other ATP-hydrolyzing wall enzymes could explain why so much higher concentrations of ATP than of ATP $\gamma \mathrm{S}$ were needed to induce growth responses.

Because ATP, and to a lesser extent, ADP, can chelate divalent cations, the mM levels of ATP and ADP used by Tang et al. [37] raise the question of whether the nucleotide-induced growth changes observed by them could be due to chelation of $\mathrm{Ca}^{2+}, \mathrm{Zn}^{2+}$, or other divalent cations known to influence growth. As discussed by the authors [37], this interpretation is rendered unlikely by their observation that the effects of ATP on gravitropism were more pronounced at $\mathrm{pH} 4.1$ than at 5.7, but the ability of ATP to chelate divalent cations is significantly weaker at the lower $\mathrm{pH}$ value. Further arguing against attributing the nucleotide effects to chelation is the authors' observation that $0.1 \mathrm{mM} \mathrm{ATP} \gamma \mathrm{S}$ is as effective as $2 \mathrm{mM}$ ATP in blocking gravitropism [37, 38]. 
In animals secretory vesicles typically contain high concentrations of ATP in their lumen and deliver this ATP into the ECM when they fuse with the plasma membrane [40, 41]. In plants growth requires the delivery of secretory vesicles to the plasma membrane and the export of their contents into the cell wall space [39]. In the case of the root, exocytotic vesicles involved in root growth could contain cell wall material, or other secretions of the root, such as root mucilage required for soil penetration. If plant secretory vesicles also contain significant ATP, there would have to be a mechanism for removing the ATP that is released into the plant wall (ECM) during cell growth in order to prevent accumulation of higher concentrations of extracellular ATP that, as discussed above, can be inhibitory to plant growth. In animals ectoapyrases (ecto-NTPDases) are among the key enzymes controlling the extracellular ATP concentration [42], and these enzymes have been documented in plants [13-18]. In this context it is noteworthy that knocking out two closely related apyrases in Arabidopsis (AtApyl and AtApy2) results in growth defects, the most dramatic of which is the failure of pollen to grow [20]. Chemical inhibitors of apyrase activity or exogenous application of $\mathrm{mM}$ concentrations of ATP also inhibit Arabidopsis pollen germination [20]. Transgenic Arabidopsis overexpressing a pea apyrase can take up the gamma phosphate from radiolabeled ATP with greater efficiency than wild-type plants, suggesting that plant ecto-apyrases can participate in the hydrolysis of extracellular ATP [19]. There are seven members of the apyrase family in Arabidopsis, and, given the prospective growth regulatory roles of extracellular ATP proposed above, it seems likely that one or more of these could function to regulate extracellular ATP concentrations in wild-type Arabidopsis as well.

\section{The search for a plant extracellular ATP receptor: A P2-like receptor or a novel receptor type?}

The means by which extracellular ATP is perceived by plant cells is currently unknown. However, the $\Delta\left[\mathrm{Ca}^{2+}\right]_{\text {cyt }}$ and superoxide responses induced by ATP both have a set of characteristics - low threshold of induction, dose dependency, substrate specificity and saturability, and inducibility by non-hydrolysable analogs of ATP- that indicate these effects are likely to be mediated by a distinct receptor (or receptors). Two structurally distinct classes of nucleotide receptors have been characterized in animal systems. These include the P2X family, which are twotransmembrane (TM) domain subunits that oligomerize to form ligand-gated ion channels, and the P2Y family of 7pass heterotrimeric G-protein linked receptors [33]. Both classes are comprised of multigene family members with modest sequence conservation between orthologues. Extensive database searches using the Hidden Markov Model have failed to identify plant homologues to either P2X or P2Y-type receptors, although it is possible that the sequence similarity is simply too low for this to be a viable approach. It is also possible that a novel receptor type exists, especially considering that yeast, bacteria and lower animals [43, 44], in addition to plants, have all been observed to respond to extracellular ATP, and in many cases, the receptors accounting for these effects have yet to be identified.

The pharmacological data of Demidchik et al. [27] and Jeter et al. [29] convincingly argue for the involvement of a cation channel through which calcium enters into the plant cell cytoplasm from the ECM (Table 1 and Figure 2). In this regard, although one or both of the cationic calcium channel blockers $\mathrm{Gd}^{3+}$ and $\mathrm{La}^{3+}$ can block the responses of the root to ATP analogues [27], and of the whole seedling to ADP analogues [29] (Figure 2B), these agents have been observed to have intracellular effects as well $[45,46]$. The antagonistic actions of $\mathrm{Gd}^{3+}$ and $\mathrm{La}^{3+}$ implicate calcium channel activity in the ATP response, however the efficacy of the calcium chelator BAPTA provides more definitive evidence that an extracellular source of the calcium ions contributes to the increased $\left[\mathrm{Ca}^{2+}\right]_{\text {cyt }}[29]$ (Figure 2B).

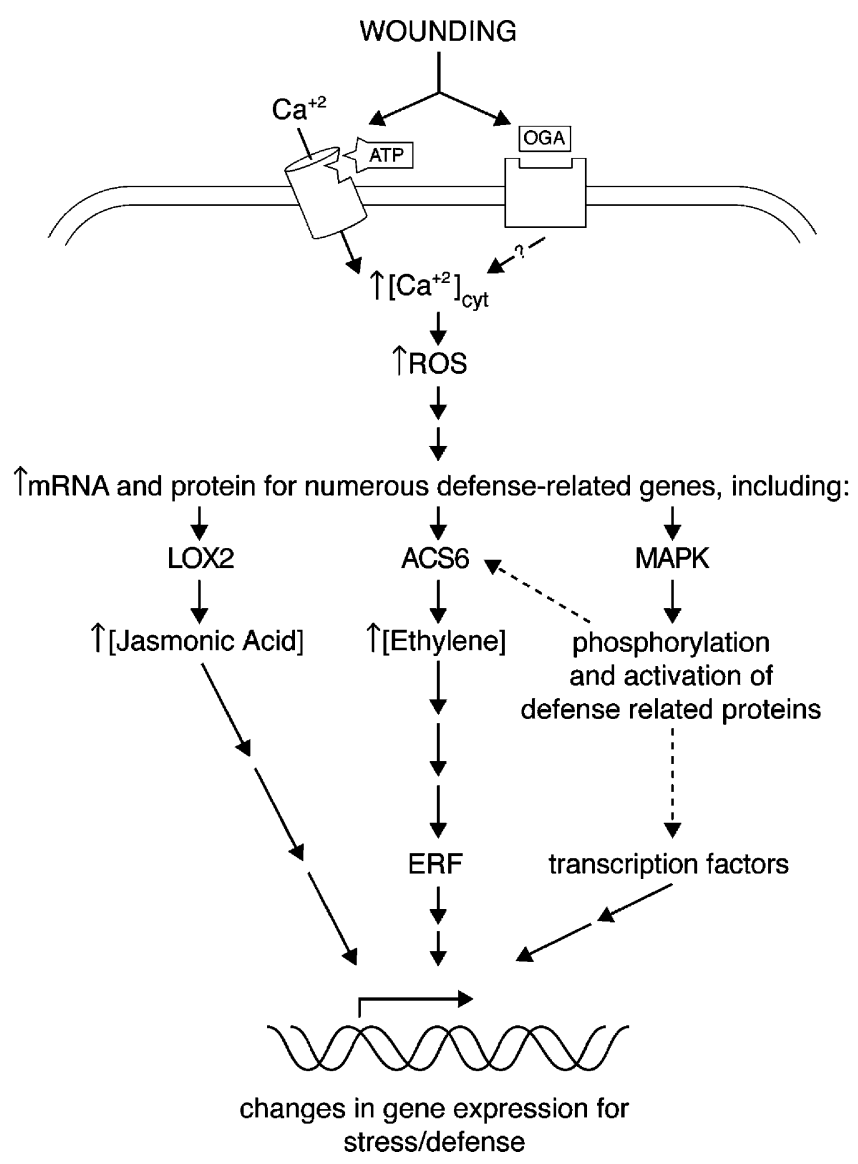

Figure 3. A proposed model showing the concerted effects of ATP and OGA on plant cell signal transduction pathways mediating stress, wound and defense responses. Wounding releases ATP and OGA into the plant cell wall space, each binding to distinct receptors. Activation of the ATP receptor leads to an influx of calcium from the ECM through a cation channel (either directly or indirectly), whereas activation of the OGA receptor leads to a release of calcium from unknown internal stores [30]. Downstream production of reactive oxygen species (ROS) could represent a point of convergence, inducing an increase in the levels of the stress hormone ethylene. MAPK family members likely interact with the signaling pathway at multiple points [48-51], and it is the concerted effects of all of these signaling mechanisms that ultimately alter gene expression. The dotted line refers to post-translational regulation of key wound and stress enzymes by MAPK activity. 
If the receptor and channel are one and the same, this type of plasma membrane localized receptor would be reminiscent of the P2X-type receptor, and the influx of calcium could contribute to the membrane depolarization measured by Lew and Dearnaley [23]. Alternatively, the activation of a distinct receptor could couple with the opening of a separate calcium channel, a possibility which cannot be eliminated given the available data. The expression of an Arabidopsis cDNA library in a heterologous system (such as Xenopus oocytes) could be a means to identify a plant extracellular ATP receptor, especially if the receptor is also the channel. Regardless, further studies are required to answer the question of how plant cells sense, and initiate responses to, extracellular ATP.

Curiously, the cytokinin 6-benzylaminopurine (6-BAP) has been reported to activate positive inotropism when applied to Wistar rat heart tissue, in a manner consistent with the action of P2-receptors [47]. If plant-derived cytokinins can cross-react with animal P2-receptors, it seems possible that in planta extracellular ATP could be stimulating cytokinin receptors. However, although cytokinin and extracellular ATP have some similar effects on plant physiology (e.g. decreased root length, root hair growth rate) at the cellular level cytokinin signaling involves plasma membrane hyperpolarization rather than the extracellular ATP induced depolarization reported by Lew and Dearnaley [23]. Also, cytokinin responses are sensitive to the anion transport inhibitor diisothiocyanostilbene$2,2^{\prime}$ disulfonic acid (DIDS) [48]. Because DIDS is also an antagonist of some P2-receptors [33] Jeter et al. [29] tested its effects on the calcium transients induced by ADP $\beta S$, and reported it to be an ineffective blocking agent. Although these data indicate that cytokinin and extracellular ATP responses are mediated by separate receptors, interactions in downstream signaling remain a distinct possibility.

\section{Perception of extracellular ATP activates downstream calcium-dependent signaling networks}

Indeed, the biological effects of extracellular ATP would necessarily interconnect with any additional signaling agents (such as ethylene, auxin and jasmonic acid) that were induced secondarily. Furthermore, other signaling molecules (e.g. OGA) could also act together with extracellular ATP to elicit cellular and physiological responses. For example, although OGA and extracellular ADP have different mechanisms to increase the $\left[\mathrm{Ca}^{2+}\right]_{\text {cyt }}$ in plant cells, both can stimulate the oxidative burst [29, 32], and both have been proposed to induce production of the stress responsive hormone ethylene [29, 49]. Protein phosphorylation cascades involving MAPK family members are likely players in the signaling networks mediating these effects, and could be involved both upstream and downstream of ethylene signaling [50-53] (Figure 3). Ultimately, it is the concerted effects of all of the environmental cues and signaling agents interacting, and in some cases converging on the same intracellular signaling pathways, that thus dictate the actual biological response. Extracellular ATP-signaling alone is insufficient to explain all of the pronounced and varied effects of stress on plant cells; however extracellular nucleotide derivatives could play an important role in mediating plant responses to stress stimuli.

\section{Evolutionary conservation}

Despite obvious differences between animals and plants, at the cellular level many similarities exist. These include the conservation of intracellular signaling mechanisms, such as the central role for the fundamental secondary messenger calcium. Even extracellular signaling molecules previously considered unique to specialized animal cells have been shown to function as cell-cell signals outside plant cells as well. For example, the classical animal neurotransmitter glutamate has been shown to induce calcium transients in plant cells [54], and glutamate receptor homologues are present in the Arabidopsis genome. Given this conservation of signaling mechanisms, it is perhaps not too surprising that both animal and plant cells appear to have evolved common mechanisms to utilize the abundant and ubiquitous intracellular metabolites ATP and ADP as extracellular signaling agents. Further studies will reveal the nature of plant cell surface receptors activated by extracellular nucleotides, and elucidate the similarities and differences between animal and plant cellular machinery sensing and responding to these cell-cell signaling agents.

\section{Acknowledgments}

The research of the authors reviewed here was supported by grants from the NSF (IBN-0344221) and NASA (NAG2-1586 and NAG10-295) to S. J. R.

\section{References}

1. Kamizyo A, Tanaka N. Studies on the generative nuclear divisions: III. Effects of exogenous ATP on the generative nuclear divisions in Lilium longiflorum. Cytologia 1982; 47: 195-205.

2. Nejidat A, Itai $\mathrm{C}$, Roth-Bejerano N. Stomatal response to ATP mediated by phytochrome. Physiol Plant 1983; 57: 367-70.

3. Jaffe MJ. The role of ATP in mechanically stimulated rapid closure of the Venus's-Flytrap. Plant Physiol 1973; 51: 17-8.

4. Bonsignore CA, Hepler PK. Caffeine inhibition of cytokinesis: Dynamics of cell plate formation-deformation in vivo. Protoplasma 1985; 129: 28-35.

5. Roberts $\mathrm{AW}$, Haigler $\mathrm{CH}$. Methylxanthines reversibly inhibit tracheary element differentiation in suspension cultures of Zinnia elegans. Planta 1992; 186: 586-92.

6. Pierson ES, Smith PJS, Shipley AM et al. $\mathrm{Ca}^{2+}$ fluxes around pollen grains and pollen tubes of lily; normal development and effect of temperature shock, BAPTA-type buffer microinjection and depletion of boric acid from the medium. Biol Bull 1993; 185: 302-3.

7. Brault M, Caiveau O, Pedron J et al. Detection of membrane-bound cytokinin-binding proteins in Arabidopsis thaliana cells. Eur J Biochem 1999; 260: 512-9.

8. Dubyak GR, El-Moatassim C. Signal transduction via P2-purinergic receptors for extracellular ATP and other nucleotides. Am J Physiol 1993; 265: C577-606.

9. Sauer H, Hescheler J, Wartenberg M. Mechanical strain-induced $\mathrm{Ca}^{2+}$ waves are propagated via ATP release and purinergic receptor activation. Am J Physiol, Cell Physiol 2000; 279: C295-307. 
10. Bowler WB, Buckley KA, Gartland A et al. Extracellular nucleotide signaling: A mechanism for integrating local and systemic responses in the activation of bone remodeling. Bone 2001; 28: 507-12.

11. Boyum R, Guidotti G. Effect of ATP binding cassette/multidrug resistance proteins on ATP efflux of Saccharomyces cerevisiae. Biochem Biophys Res Commun 1997; 230: 22-6.

12. Thomas C, Rajagopal A, Windsor B et al. A role for ectophosphatase in xenobiotic resistance. Plant Cell 2000; 12: 519-33.

13. Handa M, Guidotti G. Purification and cloning of a soluble ATPdiphosphohydrolase (apyrase) from potato tubers (Solanum tuberosum). Biochem Biophys Res Commun 1996; 218: 916-23.

14. Hsieh H, Tong C, Thomas C, Roux SJ. Light-modulated abundance of an mRNA encoding a calmodulin-regulated, chromatin-associated NTPase in pea. Plant Mol Biol 1996; 30:135-47.

15. Roberts NJ, Brigham J, Wu B et al. A nod factor-binding lectin is a member of a distinct class of apyrases that may be unique to the legumes. Mol Gen Genet 1999; 262: 261-7.

16. Steinebrunner I, Jeter C, Song C, Roux SJ. Molecular and biochemical comparison of two different apyrases from Arabidopsis thaliana. Plant Physiol Biochem 2000; 38: 1-10.

17. Day RB, McAlvin CB, Loh JT et al. Differential expression of two soybean apyrases, one of which is an early nodulin. Mol PlantMicrob Interact 2000; 13: 1053-70.

18. Cohn JR, Uhm T, Ramu S et al. Differential regulation of a family of apyrase genes from Medicago truncatula. Plant Physiol 2001; 125: 2104-19.

19. Thomas C, Sun Y, Naus K et al. Apyrase functions in plant phosphate nutrition and mobilizes phosphate from extracellular ATP. Plant Physiol 1999; 119: 543-51.

20. Steinebrunner I, Wu J, Sun Y et al. Disruption of apyrases inhibits pollen germination in Arabidopsis. Plant Physiol 2003; 131: 1638-47.

21. Kalsi G, Etzler ME. Localization of a Nod factor-binding protein in legume roots and factors influencing its distribution and expression. Plant Physiol 2000; 124: 1039-48.

22. McAlvin CB, Stacey G. Transgenic expression of the soybean apyrase in Lotus japonicus enhances nodulation. Plant Physiol 2005; 137: 1456-62.

23. Lew RR, Dearnaley JDW. Extracellular nucleotide effects on the electrical properties of growing Arabidopsis thaliana root hairs. Plant Sci 2000;153: 1-6.

24. Sanders D, Brownlee C, Harper J. Communicating with calcium. Plant Cell 1999; 11: 691-706.

25. Geurts R, Bisseling T. Rhizobium nod factor perception and signalling. Plant Cell 2002; 14(Suppl): S239-49.

26. Knight M, Read ND, Campbell A, Trewavas AJ. Imaging calcium dynamics in living plants using semi-synthetic recombinant aequorins. J Cell Biol 1993; 121: 83-90.

27. Demidchik $\mathrm{V}$, Nichols $\mathrm{C}$, Oliynyk $\mathrm{M}$ et al. Is ATP a signaling agent in plants? Plant Physiol 2003; 133: 456-61.

28. Tang W, Jeter C, Henaff E et al. Extracellular ATP acts as a potential signaling molecule in controlling growth and development in Arabidopsis. Plant Biology 2003, Abs \# 1432. (http://abstracts.aspb. org/pb2003/public/P72/1467.html).

29. Jeter $\mathrm{C}$, Tang $\mathrm{W}$, Henaff $\mathrm{E}$ et al. Evidence of a novel cell signaling role for extracellular adenosine triphosphates and diphosphates in Arabidopsis. Plant Cell 2004; 16: 2652-64.

30. Chandra S, Stennis M, Low PS. Measurement of $\mathrm{Ca}^{2+}$ fluxes during elicitation of the oxidative burst in aequorin-transformed tobacco cells. J Biol Chem 1997; 272: 28274-80.

31. Apel K, Hirt H. Reactive oxygen species: Metabolism, oxidative stress, and signal transduction. Annu Rev Plant Biol 2004; 55: 373-99.

32. Song C, Wang X, Stout S, Roux SJ. A role for extracellular ATP and apyrases in regulating wound responses in leaves of Arabidopsis thaliana. Plant Biology 2005, Abstract \# 54. (http://abstracts.aspb. org/pb2005/public/P34/7518.html).
33. Ralevic V, Burnstock G. Receptors for purines and pyrimidines. Pharm Rev 1998; 50: 413-92.

34. Mizoguchi T, Irie K, Hirayama $\mathrm{T}$ et al. A gene encoding a mitogenactivated protein kinase kinase kinase is induced simultaneously with genes for a mitogen-activated protein kinase and an S6 ribosomal protein kinase by touch, cold and water stress in Arabidopsis thaliana. Proc Natl Acad Sci USA 1996; 93: 765-9.

35. Reymond P, Weber H, Damond M, Farmer EE. Differential gene expression in response to mechanical wounding and insect feeding in Arabidopsis. Plant Cell 2000; 12: 707-19.

36. Morgan PW, Drew MC. Ethylene and plant responses to stress. Physiol Plant 1997; 100: 620-30.

37. Tang WQ, Brady SR, Sun Y et al. Extracellular ATP inhibits root gravitropism at concentrations that inhibit polar auxin transport. Plant Physiol 2003; 131: 147-54.

38. Tang WQ. Effects of extracellular ATP and ADP on growth and development of Arabidopsis seedlings. PhD dissertation, The University of Texas at Austin 2004.

39. Clark G, Lee D, Dauwalder M, Roux SJ. Immunolocalization and histochemical evidence for the association of two different Arabidopsis annexins with secretion during early seedling growth and development. Planta 2005; 220: 621-31.

40. Joseph SM, Buchakjian MR, Dubyak GR. Colocalization of ATP release sites and ecto-ATPase activity at the extracellular surface of human astrocytes. J Biol Chem 2003; 278: 23331-42.

41. Zhong X, Malhotra R, Guidotti G. ATP uptake in the golgi and extracellular release require Mcd4 protein and the vacuolar $\mathrm{H}-$ ATPase. J Biol Chem 2003; 278: 33436-44.

42. Alvarado-Castillo C, Harden TK, Boyer JL. Regulation of P2Y(1) receptor-mediated signaling by the ectonucleoside triphosphate diphosphohydrolase isozymes NTPDase1 and NTPDase2. Mol Pharmacol 2005; 67: 114-22.

43. Burnstock G. Purinoceptors: Ontogeny and phylogeny. Drug Dev. Res 1996; 39: 204-42.

44. Hennessey TM. Responses of the ciliates Tetrahymena and Paramecium to external ATP and GTP. Purinergic Signalling 2005; 1: 101-10.

45. Polisensky DH, Braam J. Cold-shock regulation of the Arabidopsis TCH genes and the effects of modulating the intracellular calcium levels. Plant Physiol 1996; 1271-79.

46. Cessna SG, Low PS. Activation of the oxidative burst in aequorintransformed Nicotinia tabacum cells is mediated by protein kinaseand anion channel-dependent release of $\mathrm{Ca}^{2+}$ from internal stores. Planta 2001; 214: 126-34.

47. Froldi G, Gallo U, Ragazzi E, Caparrotta L. 6-Benzylaminopurine: A plant derived cytokinin inducing positive inotropism by P2-purinoceptors. Planta Med 1999; 65: 245-9.

48. Silverman FP, Assiamah AA, Bush DS. Membrane transport and cytokinin action in root hairs of Medicago sativa. Planta 1998; 205: 23-31.

49. Rojo E, Solano R, Sanchez-Serrano JJ. Interactions between signaling compounds involved in plant defense. J Plant Growth Regul 2003; 22: 82-98.

50. Kim CY, Liu Y, Thorne ET et al. Activation of a stress-responsive mitogen-activated protein kinase cascade induces the biosynthesis of ethylene in plants. Plant Cell 2003; 15: 2707-18.

51. Ouaked F, Rozhon W, Lecourieux D, Hirt H. A MAPK pathway mediates ethylene signaling in plants. EMBO J 2003; 22: 1282-8.

52. Liu YD, Zhang SQ. Phosphorylation of 1-aminocyclopropane-1carboxylic acid synthase by MPK6, a stress-responsive mitogenactivated protein kinase, induces ethylene biosynthesis in Arabidopsis. Plant Cell 2004; 12: 3386-99.

53. Cheong YH, Moon BC, Kim JK et al. BWMK1, a rice mitogenactivated protein kinase, locates in the nucleus and mediates pathogenesis-related gene expression by activation of a transcription factor. Plant Physiol 2003;132: 1961-72.

54. Dennison KL, Spalding EP. Glutamate-gated calcium fluxes in Arabidopsis. Plant Physiol 2000; 124: 1511-4. 